\title{
Game Analysis of Photovoltaic Enterprise Competitive Strategy Based on Technology Innovation
}

\author{
Geng Hejiang ${ }^{1, a}$, Zhang Renjun ${ }^{2, b}$ \\ ${ }^{1,2}$ Business School, Hohai University Nanjing, China \\ aemail: genghejiang@163.com, bemail: rjzhang@hhu.edu.cn
}

Keywords: photovoltaic enterprise; technology innovation; competitive strategy; game analysis

\begin{abstract}
Analysis of model shows that technology innovation provides photovoltaic product additional value, increases photovoltaic product differentiation and reduces consumers' price sensitivity, but enterprise profit is decided by the efficiency of technology innovation, only the high efficiency of technology innovation can increase enterprise competence. Finally, the paper gives the strategy advices on technology innovation of photovoltaic enterprise.
\end{abstract}

\section{Introduction}

With the fossil energy shrinking gradually and the environment, seriously deteriorating, renewable energy is receiving more and more attention. After the accident of Fukushima nuclear power station in Japan on March 11, 2011, solar photovoltaic power generation is attached importance to by all countries because of its safety and pollution-free. Scholars are more bullish on photovoltaic industry prospects. S.R. Wenham (2001) et al. thinks that photovoltaic industry plays an important role in economic development, and technology innovation is the key to photovoltaic industry development ${ }^{[1]}$. H. Winfried (2006) predicts the trend of the global photovoltaic market, and puts forward the technology paradigm and the measures of the product market development ${ }^{[2]}$. T.A. Stijn et al(2009) analyzes the concept of the photovoltaic industrialization by an empirical way, and thinks that technology innovation is an important driving force of the rapid development of photovoltaic industry ${ }^{[3]}$. A. Keller et al (2009) think that investment cost of photovoltaic cells goes up due to technology reasons, and the solar photovoltaic power generation incurs cost disadvantage [4]. Wang Faming (2009) studies from the value chain perspective that technology level and resource scarcity of photovoltaic industry decide industry barriers, profits and distribution characteristics $^{[5]}$. Zhao Yuwen (2010) points out that the regulations and policy are the most impetus of photovoltaic industry development, and implementation of the law of electricity price can pull the development of technology quickly, and reduce the cost of the industry ${ }^{[6]}$.

The Existing studies concentrate on the macro or industry level to analyze it qualitatively and give some advice. The paper explains that the product homogeneity is the reason of low price competition between photovoltaic enterprises, and studies photovoltaic enterprise how to realize its product value increasing by technology innovation based on the analysis of duopoly model.

\section{Game Model Assumption and Analysis}

The paper studies the competition strategy of technology innovation of photovoltaic enterprise based on Bertrand model.

\section{Model Basic Assumptions}

We assume that there are only two photovoltaic enterprises in the market, and their products are alternative each other, and the price of one of them will influence the price and product of the other.

The total output in the market is the sum of two photovoltaic enterprises' product, namely: $Q=q_{1}+q_{2}$, and total demand is equal to total yield; $q_{i}$ is product or demand of market of photovoltaic enterprise $i$.

The fixed cost is not taken into consideration, and two enterprises have the same unit marginal $\operatorname{cost} c$.

$\mathrm{R}$ is enterprise profit and the function of product $\mathrm{Q}$. 
Technology innovation of enterprise can increase production value $v$ and need to invest innovation $\operatorname{cost} c_{0}$.

$\beta$ represents product differentiation degree, and $0<\beta<1, \beta \rightarrow 0$ means that enterprise products are completely different, $\beta \rightarrow 1$ means that enterprises products are no difference. $p_{i}$ is product price of photovoltaic enterprise $i$. $a$ is the highest price, and $b$ is the demand elasticity of price to market. $a$ and $b$ are both positive.

According to Bowley duopoly game model, inverse demand functions of photovoltaic enterprise 1 and 2 are following respectively.

$$
p_{1}=a-b\left(q_{1}+\beta q_{2}\right), \quad p_{2}=a-b\left(\beta q_{1}+q_{2}\right)
$$

And demand functions respectively:

$$
q_{1}=\frac{a(1-\beta)-p_{1}+\beta p_{2}}{b\left(1-\beta^{2}\right)}, \quad q_{2}=\frac{a(1-\beta)-p_{2}+\beta p_{1}}{b\left(1-\beta^{2}\right)}
$$

From formulae (1) and (2), we can get the utility function of photovoltaic enterprise $i$.

\section{Game Model Analysis}

$$
U_{i}=q_{i}\left(p_{i}-c\right), \quad(i=1,2)
$$

Two photovoltaic enterprises implement game, and the aim of game is to maximize their respective profits. Combine formulae (1) and (2), and solve first order condition of formula (3).

$$
p_{1}^{*}=\frac{a(1-\beta)+\beta p_{2}+c}{2}, \quad p_{2}^{*}=\frac{a(1-\beta)+\beta p_{1}+c}{2}
$$

From formulae (4), we can obtain product equilibrium price of two photovoltaic enterprises.

$$
p_{1}^{*}=p_{2}^{*}=\frac{a(1-\beta)+c}{2-\beta}
$$

From formulae (2) and (5), we can obtain product equilibrium yield of two enterprises.

$$
q_{1}^{*}=q_{2}^{*}=\frac{a-c}{b(1+\beta)(2-\beta)}
$$

Therefore, we can get the decision of two enterprises on the condition of equilibrium.

$$
p_{1}^{*}=p_{2}^{*}=\frac{a(1-\beta)+c}{2-\beta}, \quad q_{1}^{*}=q_{2}^{*}=\frac{a-c}{b(1+\beta)(2-\beta)}
$$

If $\beta \rightarrow 1$, then $p_{1}^{*}=p_{2}^{*} \rightarrow c$, means that the product prices of two photovoltaic enterprises are close to the marginal cost, and profit of enterprise is close to zero. The products of two photovoltaic enterprises strictly tend to homogeneity. Therefore, the only result of game between two photovoltaic enterprises is to lower product price. This will result in excessive competition. It can fully explain the reason on the low price competition of photovoltaic enterprises.

\section{Technology Innovation Model Offering Extra Value \\ Model Basic Assumptions}

We assume photovoltaic enterprise 1 obtains product extra value by technology innovation, but enterprise 2 has not implemented technology innovation. Then the product prices and demands of two photovoltaic enterprises are both influenced by the innovation. $c_{0}$ is the investment cost of photovoltaic enterprise 1 for extra value. $v$ is the extra value of photovoltaic enterprise 1 product by technology innovation. Now we can regard $e=\frac{v}{c_{0}}$ as efficiency of technology innovation.

Then demand functions of photovoltaic enterprise 1 and 2 can be following.

$$
\bar{q}_{1}=\frac{a(1-\beta)-p_{1}+\beta\left(p_{2}+v\right)}{b\left(1-\beta^{2}\right)}, \bar{q}_{2}=\frac{a(1-\beta)-\left(p_{2}+v\right)+\beta p_{1}}{b\left(1-\beta^{2}\right)}
$$

Two photovoltaic enterprises implement duopoly game, and then we can get the decisions of product price and yield of photovoltaic enterprise 1 and 2 on the condition of equilibrium as follow.

$$
\bar{p}_{1}^{*}=\frac{a(1-\beta)+c}{2-\beta}+\frac{\beta v+2 c_{0}}{4-\beta^{2}}, \quad \bar{q}_{1}^{*}=\frac{a-c}{b(1+\beta)(2-\beta)}+\frac{\beta v+c_{0}\left(\beta^{2}-2\right)}{b\left(1-\beta^{2}\right)\left(4-\beta^{2}\right)} ;
$$




$$
\bar{p}_{2}^{*}=\frac{a(1-\beta)-v+c}{2}+\frac{\beta[a(1-\beta)+c]}{2(2-\beta)}+\frac{\beta\left(\beta v+2 c_{0}\right)}{2\left(4-\beta^{2}\right)}, \quad \bar{q}_{2}^{*}=\frac{a-c}{b(1+\beta)(2-\beta)}+\frac{v\left(\beta^{2}-2\right)+\beta c_{0}}{b\left(1-\beta^{2}\right)\left(4-\beta^{2}\right)}
$$

\section{Analysis on Model of Technology Innovation}

Technology innovation promotes the product price of photovoltaic enterprise 1, and the market demands and prices of photovoltaic enterprises 1 and 2 depend on technology innovation efficiency $e$ of photovoltaic enterprise 1 too.

Analysis of the price decision of photovoltaic enterprise 1. $p_{1}^{*}<\bar{p}_{1}^{*}$, namely, $p_{1}^{*}-\bar{p}_{1}^{*}=\frac{\beta v+2 c_{0}}{4-\beta^{2}}=\frac{c_{0}(\beta e+2)}{4-\beta^{2}}<0$. The conclusion shows that photovoltaic enterprise 1 raises its price by technology innovation to reduce the consumer sensitive. The higher technology innovation efficiency is, the more photovoltaic product price increment is. The formula judgment is $\bar{p}_{1}^{*}-p_{1}^{*}-c_{0}=\frac{\beta v+\beta^{2} c_{0}-2 c_{0}}{4-\beta^{2}}$, if $\bar{p}_{1}^{*}-p_{1}^{*}-c_{0} \geq 0$, it means that the technology innovation of photovoltaic enterprise 1 increases profits and technology innovation is valuable. If $\bar{p}_{1}^{*}-p_{1}^{*}-c_{0}<0$, it shows that technology innovation of photovoltaic enterprise 1 increases the product price, but the price increment is not sufficient to cover the marginal cost of technology innovation, and technology innovation cannot produce profits. By analyzing $\frac{\beta v+\beta^{2} c_{0}-2 c_{0}}{4-\beta^{2}}$, we know that $e_{1}^{*}=\frac{2-\beta^{2}}{\beta}$ is the boundary of the investment decision of photovoltaic enterprise 1 .

Analysis of the price decision of photovoltaic enterprise 2. $p_{2}^{*}$ and $\bar{p}_{2}^{*}$ depend on the technology innovation efficiency of photovoltaic enterprise 1 , and the boundary is $e_{2}^{*}=\frac{\beta}{2-\beta^{2}}$. If $e \leq e_{2}^{*}$, $p_{2}^{*} \leq \bar{p}_{2}^{*}$, but $\bar{p}_{2}^{*}-p_{2}^{*}<\bar{p}_{1}^{*}-p_{1}^{*}$; if $e>e_{2}^{*}, p_{2}^{*}>\bar{p}_{2}^{*}$.

The conclusion shows that the technology innovation behavior of photovoltaic enterprise 1 has certain externalities. When the technology innovation efficiency of photovoltaic enterprise 1 is $e \leq e_{2}^{*}$, its product price increases, but the price increment cannot make up for the investment cost. At the same time, product price of photovoltaic enterprise 2 increases too, but the increment is less than that of photovoltaic enterprise 1 . When the technology innovation efficiency is $e_{2}^{*}<e<e_{1}^{*}$, the product price of enterprise 1 raises, similarly the increment is less than the cost, but the product price of enterprise 2 decreases. When the technology innovation efficiency is $e \geq e_{1}^{*}$, the product price of enterprise 1 raises and the increment is sufficient to cover the cost, but the product price of enterprise 2 continues to decrease.

Analysis of the product decision of photovoltaic enterprise $1 . q_{1}^{*}$ and $\bar{q}_{1}^{*}$ depends on the technology innovation efficiency of photovoltaic enterprise 1 , if $e \geq e_{1}^{*}, q_{1}^{*} \leq \bar{q}_{1}^{*}$; if $e<e_{1}^{*}$, $q_{1}^{*}>\bar{q}_{1}^{*}$, this means that the market demand of photovoltaic enterprise 1 product is related to its technology innovation efficiency. If $e \geq e_{1}^{*}$, the product demand of photovoltaic enterprise 1 increases, namely $\Delta q_{1} \geq 0$. If $e<e_{1}^{*}$, the product demand of photovoltaic enterprise 1 decreases, namely $\Delta q_{1}<0$.

Analysis of product decision of photovoltaic enterprise 2.

$q_{2}^{*}$ and $\bar{q}_{2}^{*}$ depend on the technology innovation efficiency of photovoltaic enterprise 1 too. If $e \leq e_{2}^{*}, \quad q_{2}^{*} \leq \bar{q}_{2}^{*}$; if $e>e_{2}^{*}, \quad q_{2}^{*}>\bar{q}_{2}^{*}$, this means that the market demand of photovoltaic enterprise 2 product is related to technology innovation efficiency of photovoltaic enterprise 1 , if $e \leq e_{2}^{*}$, the product demand of photovoltaic enterprise 2 increases, namely $\Delta q_{2} \geq 0$, If $e>e_{2}^{*}$, the product demand of photovoltaic enterprise 2 decreases, namely $\Delta q_{2}<0$. 
From the above analysis, we know that, only while $e_{1}^{*} \geq \frac{2-\beta^{2}}{\beta}$, the price increment of photovoltaic enterprise 1 can make up for the cost of technology innovation. Therefore, $e_{1}^{*}=\frac{2-\beta^{2}}{\beta}$ is the investment boundary of technology innovation of photovoltaic enterprise 1 . The same investment $c_{0}$ can bring higher price increment and produce more profits, if $e$ of technology innovation efficiency is higher. We can calculate the boundary of photovoltaic enterprise 2, which is influenced by technology innovation of photovoltaic enterprise $1: e_{2}^{*}=\frac{\beta}{2-\beta^{2}}$.

Technology innovation can avoid the low price competition of photovoltaic enterprise. While the technology innovation efficiency is $e \geq e_{1}^{*}$, it can produce extra value.

\section{Proposals for Competition Strategy of Photovoltaic Enterprise Technology Innovation}

Technology innovation can enlarge the product differentiation and increase profit. Based on above analysis, the paper proposes the following competition strategies on technology innovation of photovoltaic enterprise.

\section{The Differentiation Aim of Technology Innovation}

Photovoltaic enterprise has to set a differentiation aim, if it wants to obtain the sustainable competition advantage. Because the market decides the density of enterprise, if enterprise cannot offer consumers extra value, enterprise will naturally plunge into a vicious cycle of low price competition.

\section{Promoting Technology Innovation Efficiency}

Technology innovation efficiency reflects the comparative relation of the benefits and costs. Therefore, when one enterprise increases technology innovation investment, it should have the ability to improve the technology innovation efficiency, and to increase product differentiation degree to provide consumers with more extra value.

\section{Establishing Technology Innovation Mechanism}

Building mechanism of technology innovation is the fundamental measures in the competition. Government should establish the responsibility mechanism of technology innovation, investment mechanism, the participation mechanism of technology innovation and the evaluation mechanism of enterprise technology innovation, etc.

\section{Cultivating Enterprise Innovation Culture}

If mechanism is the external requirement of technology innovation, innovation culture is the inner driving force of technology innovation. After setting up creative culture of photovoltaic enterprise, you can really mobilize the innovation enthusiasm of all the enterprise members, and make the enterprise to obtain continuous innovation drive.

\section{REFERENCES}

[1] S.R.Wenham, C.B.Honsberg, J.E.Cotter, etal., "Australian educational and research opportunities arising through rapid growth in the photovoltaic industry. "Solar Energy Material \& Solar Cells, vol.67, no.1-4, pp. 647-656, 2001.

[2] H.Winfried, "PV solar electricity industry: Market growth and perspective." Solar Energy Materials \& Solar Cells, vol.90, no.18/19, pp. 3285-3311, 2006.

[3] T.A.Stijn, C.J.M.Jeroen, "Multilevel assessment of diversity, innovation and selection in the Solar Photovoltaic Industry. "Structural Change and Economic Dynamics, vol. 20, no. 1, pp. 50-60, Jan. 2009.

[4] A.Keller, Ploss, etal., ICIS Chemical Business., vol. 276, no. 4, pp. 32-33. Apr. 2009.

[5] Wang Faming, "the Study on Chinese photovoltaic industry upgrading under the condition of global value chain." Economic management journal,vol. 31, no. 11, pp. 55-61, Nov. 2009.

[6] Zhao Yuwen, "Photovoltaic industry strategy prospects and policy thinking." Energy Saving of Non-Ferrous Metallurgy, no. 12, pp.1-5, Dec. 2010. 